\title{
The Effect of Dolutegravir on the Pharmacokinetics of Metformin in Healthy Subjects
}

\author{
Ivy H. Song, PhD, * Jian Zong, PhD, * Julie Borland, BPharm, * Fred Jerva, PhD, * \\ Brian Wynne, MD, † Maciej J. Zamek-Gliszczynski, PhD, * Joan E. Humphreys, MS,* \\ Gary D. Bowers, PhD,* and Mike Choukour, PhD $\neq$
}

\begin{abstract}
Background: Dolutegravir is an integrase strand transfer inhibitor (INSTI) licensed for use in HIV-1 infection and is an inhibitor of organic cation transporter 2 (OCT2). This study assessed the effect of dolutegravir on the pharmacokinetics of metformin, an OCT2 substrate.

Design: This was an open-label, parallel-group, 3-period crossover study in healthy adult subjects. Subjects were enrolled into 1 of 2 treatment cohorts ( 15 subjects/cohort) receiving metformin $500 \mathrm{mg} \mathrm{q1} 2 \mathrm{~h}$ for 5 days in period 1; metformin $500 \mathrm{mg}$ q12h plus dolutegravir $50 \mathrm{mg}$ $\mathrm{q} 24 \mathrm{~h}$ (cohort 1) or $50 \mathrm{mg}$ q12h (cohort 2) for 7 days in period 2; and metformin $500 \mathrm{mg} \mathrm{q} 12 \mathrm{~h}$ for 10 days in period 3 . There were no washout periods between treatments. Effects of dolutegravir on metformin transport and paracellular permeability were evaluated in vitro.
\end{abstract}

Results: Co-administration of dolutegravir $50 \mathrm{mg}$ q24h increased metformin area under the curve $(0-\tau)$ by $79 \%$ and Cmax by $66 \%$, whereas dolutegravir $50 \mathrm{mg} \mathrm{q} 12 \mathrm{~h}$ increased metformin area under the curve $(0-\tau)$ and $\mathrm{Cmax}$ by $145 \%$ and $111 \%$, respectively. Metformin $t(1 / 2)$ remained unchanged. Increased metformin exposure during dolutegravir co-administration returned to period 1 levels after dolutegravir discontinuation in period 3. Co-administration of dolutegravir and metformin was well tolerated. In vitro, dolutegravir was not a clinically relevant inhibitor of OCT1, OCT3, multidrug and toxin extrusion protein 1 , multidrug and toxin extrusion protein $2-\mathrm{K}$, or plasma membrane monoamine transporter, and it did not

Received for publication August 21, 2015; accepted February 26, 2016.

From the *GlaxoSmithKline, Research Triangle Park, NC; $\uparrow$ ViiV Healthcare, Collegeville, PA; and †ेParexel, Billerica, MA.

This study was funded by ViiV Healthcare.

Presented in part at the HIV Drug Therapy Glasgow Congress, November 2-6, 2014, Glasgow, United Kingdom.

I.H.S., J.Z., J.B., F.J., B.W., M.J.Z.-G., J.E.H., and G.D.B. are employees and shareholders of GlaxoSmithKline. M.C. is a contracted worker for GlaxoSmithKline. All listed authors meet the criteria for authorship set forth by the International Committee of Medical Journal Editors. The authors attest that the funding source did not have an influence on the analysis and reporting of results.

Supplemental digital content is available for this article. Direct URL citations appear in the printed text and are provided in the HTML and PDF versions of this article on the journal's Web site (www.jaids.com).

ClinicalTrials.gov identifier: NCT02064374.

Correspondence to: Brian Wynne, MD, ViiV Healthcare, Mail Stop UP4410, Collegeville, PA 19426 (e-mail: brian.r.wynne@viivhealthcare.com).

Copyright (C) 2016 Wolters Kluwer Health, Inc. All rights reserved. This is an open access article distributed under the terms of the Creative Commons Attribution-NonCommercial-NoDerivatives License 4.0 (CC BY-NC$\mathrm{ND}$ ), which permits downloading and sharing the work provided it is properly cited. The work cannot be changed in any way or used commercially. affect metformin paracellular permeability or uptake into an intestinal cell line.

Conclusions: Dolutegravir significantly increased metformin plasma exposure, which can be partially explained by OCT2 inhibition. It is recommended that dose adjustments of metformin be considered to maintain optimal glycemic control when patients are starting/stopping dolutegravir while taking metformin.

Key Words: dolutegravir, metformin, drug interaction, pharmacokinetics

(J Acquir Immune Defic Syndr 2016;72:400-407)

\section{INTRODUCTION}

Dolutegravir (Tivicay; ViiV Healthcare, Research Triangle Park, NC) belongs to a novel class of antiretroviral drugs known as integrase strand transfer inhibitors (INSTIs) and blocks the activity of the HIV viral integrase enzyme, preventing the insertion of the viral genome into the host DNA. Dolutegravir is well tolerated, has low to moderate pharmacokinetic (PK) variability, and has sustained antiviral activity when combined with other antiretroviral therapy (ART), without the need for PK boosting, in treatmentnaive, treatment-experienced but INSTI-naive, and treatmentexperienced and INSTI-resistant adults. ${ }^{1-6}$ Dolutegravir is currently approved at 2 dose levels, $50 \mathrm{mg}$ once daily (for INSTI-naive subjects) and $50 \mathrm{mg}$ twice daily (for INSTIresistant subjects).

Metformin (Glucophage; Bristol-Myers Squibb Company, Princeton, NJ), an antihyperglycemic agent that improves glucose tolerance in subjects with type 2 diabetes mellitus (T2DM) by lowering both basal and postprandial plasma glucose, is considered to be one of the best treatment options for T2DM and has been used for a number of the metabolic comorbidities that are associated with HIV. ${ }^{7}$

Under fasting conditions, the absolute bioavailability of a $500 \mathrm{mg}$ metformin immediate release (IR) tablet is approximately $50 \%-60 \% .^{8}$ Due to its highly hydrophilic nature, metformin is unable to passively diffuse across membranes, and intestinal absorption is paracellular., ${ }^{9,10}$ Although metformin can enter enterocytes by apical carrierfacilitated transport mediated primarily by organic cation transporter (OCT) 1 and plasma membrane monoamine transporter (PMAT), enterocytes lack basolateral transporters capable of secreting metformin into blood. Therefore, 
metformin uptake into enterocytes only enhances intestinal drug retention and ultimately paracellular absorption by enabling drug cycling between enterocytes and gut lumen according to the cell-gut lumen concentration gradient. ${ }^{9}$ Likewise, metformin relies on carrier-mediated uptake for distribution into organs of pharmacologic activity: OCT1 in liver and OCT3 in skeletal muscle. ${ }^{10}$ After both oral and intravenous administration, metformin does not undergo either hepatic metabolism or biliary excretion. Rather, $90 \%$ of the drug is excreted unchanged in the urine within 24 hours. Renal clearance occurs both by glomerular filtration and active tubular secretion; the latter is mediated by OCT2 uptake into tubular cells followed by secretion into urine by multidrug and toxin extrusion protein (MATE) 1 and MATE2-K. ${ }^{8,11}$ Cimetidine, a known inhibitor of MATE1 and OCT2, increased metformin peak concentration by $60 \%$ and area under the curve (AUC) by $40 \%$; therefore, careful patient monitoring and dose adjustment of metformin are recommended. ${ }^{12}$

Dolutegravir is primarily metabolized by uridine diphosphate glucuronosyl transferase $1 \mathrm{~A} 1$, with some contribution from cytochrome P450 3A4, but it is not a clinically relevant inhibitor or inducer of cytochrome P450 enzymes, an inhibitor of uridine diphosphate glucuronosyl transferase enzymes, or an inhibitor of many transporters, including OCT $1 .{ }^{13}$ Dolutegravir is a clinically relevant inhibitor of OCT $2^{14}$ and it causes elevations in serum creatinine levels through inhibition of active tubular secretion, a marker of renal OCT2/MATE inhibition. ${ }^{15}$ Thus, dolutegravir may increase plasma concentrations of drugs eliminated through OCT2/MATE, such as metformin. This study was conducted to determine if dolutegravir, administered at $50 \mathrm{mg}$ once and twice daily, affected the steady-state PK of metformin to provide guidance on coadministration. In vitro studies were also performed to evaluate the effect of dolutegravir on transporters, including PMAT, OCT3, MATE-1, and MATE2-K, as well as on the paracellular permeability of metformin.

\section{METHODS}

\section{Study Design}

This was a phase I, open-label, parallel-group, 3-period, fixed-sequence study to evaluate the effect of dolutegravir on the steady-state PK of metformin and on the safety and tolerability of metformin. The study was conducted between February 2014 and May 2014.

Eligible subjects without T2DM were sequentially assigned in a $1: 1$ ratio into 1 of 2 cohorts. In period 1, subjects in both cohorts received $500 \mathrm{mg}$ metformin IR $\mathrm{q} 12 \mathrm{~h}$ for 5 days. In period 2, subjects received $500 \mathrm{mg}$ metformin IR q12h plus either $50 \mathrm{mg}$ dolutegravir q24h (cohort 1) or $50 \mathrm{mg}$ dolutegravir q12h (cohort 2) for 7 days. In period 3, subjects in both cohorts received $500 \mathrm{mg}$ metformin IR q12h for 10 days. There was no washout between treatment periods, and all doses of study medication were taken after a moderate-fat meal. Given that the primary objective of the study was to examine the effect of dolutegravir on metformin PK, the usual starting dose of $500 \mathrm{mg}$ twice daily of metformin was used in this healthy subject study. ${ }^{12}$ Metformin was administered with a moderate-fat meal (approximately 30\% fat) to reduce gastrointestinal side effects.

\section{Study Population}

Before study initiation, the study protocol, amendments, and consent forms were reviewed and approved by the institutional review boards for the study site (Quintiles Early Clinical Development, Overland Park, KS), and all subjects provided signed consent. The study was conducted in accordance with the ethical standards of the Declaration of Helsinki and its amendments, consistent with good clinical practices and local regulatory requirements.

Healthy male or female subjects were eligible for inclusion in this study based on the following criteria: between the ages of 18 and 65 years (inclusive); body weight $\geq 50 \mathrm{~kg}$ for males and $\geq 45 \mathrm{~kg}$ for females; body mass index within the range of $18.5-31.0 \mathrm{~kg} / \mathrm{m}^{2}$ (inclusive); healthy judged on the basis of physical examination, medical history, 12-lead electrocardiogram (ECG), and laboratory testing; alanine aminotransferase (ALT), alkaline phosphatase, and bilirubin $\leq 1.5$ times the upper limit of normal; and capable of giving written informed consent. Female subjects were eligible if they were of non-child-bearing potential, of child-bearing potential with negative pregnancy test results, and agreed to use one of the protocol-defined contraception methods.

Subjects were excluded from the study if they had a chronic history of liver disease or known hepatic/biliary abnormalities (except for Gilbert's syndrome or asymptomatic gallstones); were HIV positive; were current illicit drug users or had a positive prestudy drug/alcohol screen; regularly used tobacco- or nicotine-containing products within 6 months of screening; were pregnant or lactating; had a history of regular alcohol consumption ( $>14$ drinks/wk for males and $>7$ drinks/wk for females); or tested positive for either hepatitis B or hepatitis $\mathrm{C}$ virus.

Subjects were to refrain from consuming red wine, Seville oranges, grapefruits, or grapefruit juice for 7 days before the first dose of study medication until after the final dose. Subjects were to abstain from caffeine- or xanthinecontaining products (eg, coffee, tea, cola drinks, and chocolate) and alcohol for 24 hours before the start of dosing until collection of the final PK sample during each session. The use of tobacco products was not allowed from screening until after the final follow-up visit.

\section{Safety Monitoring}

Safety was assessed throughout the study and included adverse event (AE) monitoring, clinical laboratory testing, vital sign measurements, physical examinations, and ECGs. Adverse events and laboratory events were graded according to the Division of AIDS Table for Grading the Severity of Adult and Pediatric Adverse Events Version 1.0 (December 2004, August 2009 clarification). ${ }^{16}$ 


\section{Pharmacokinetic Sampling}

Blood samples $(2 \mathrm{~mL})$ for metformin plasma PK were collected before the morning dose (within 15 minutes of dosing) and at $0.5,1,1.5,2,3,4,6,8,10$, and 12 hours postdose on the last day of each dosing period. Blood samples (2 $\mathrm{mL})$ for dolutegravir plasma PK were collected predose (within 15 minutes of dosing) and at 1, 2, 3, 4, 6, 8, and 12 hours postdose on day 7 of period 2 .

\section{Bioanalytical Methods Metformin}

Plasma samples were analyzed for metformin by Pharmaceutical Product Development (Richmond, VA) using a validated analytical method based on solid phase extraction, followed by high performance liquid chromatography with tandem mass spectrometry analysis. The lower and upper limits of quantification for metformin were 2.00 and 2000 $\mathrm{ng} / \mathrm{mL}$, respectively.

\section{Dolutegravir}

Plasma samples were analyzed for dolutegravir by Pharmaceutical Product Development (Middleton, WI) using a validated analytical method based on solid phase extraction, followed by high performance liquid chromatography with tandem mass spectrometry analysis. The lower and upper limits of quantification for dolutegravir were 20.0 and $20,000 \mathrm{ng} / \mathrm{mL}$, respectively.

\section{Pharmacokinetic Analysis}

Plasma metformin and dolutegravir concentration-time data were analyzed by noncompartmental methods using Model 200 (for extravascular administration) of Phoenix WinNonlin version 6.3 (Pharsight Corporation, St. Louis, MO). Calculations were based on the actual sampling times recorded during the study. From the plasma concentration-time data, the following PK parameters were determined, as data permitted, for metformin: maximum concentration (Cmax), time to Cmax (tmax), AUC for a dosing interval [AUC $(0-\tau)]$, half-life $[\mathrm{t}(1 / 2)]$, and apparent clearance after oral dosing $(\mathrm{CL} / \mathrm{F})$. The following pharmacokinetic parameters were determined for dolutegravir: Cmax, tmax, $\mathrm{AUC}(0-\tau), \mathrm{CL} / \mathrm{F}$, predose concentration $(\mathrm{C} 0)$, and concentration at the end of the dosing interval $(\mathrm{C} \tau)$.

\section{Statistical Analysis}

This study was designed to estimate the effect of dolutegravir on steady-state PK of metformin. No formal hypothesis was tested.

An analysis of variance, considering treatment as a fixed effect and subject as a random effect, was performed using Statistical Analysis Software (SAS, Version 9 or higher) Mixed Linear Models procedure to compare the plasma metformin PK parameters between the test treatment [dolutegravir + metformin (ie, end of period 2)] and reference treatment [metformin alone (ie, end of period 1)] for each cohort. The statistical analysis was performed on the log-transformed plasma PK parameters. Point estimates and their associated $90 \%$ confidence intervals (CIs) were con- structed for the differences between test and reference treatments for each cohort. The point estimates and their associated $90 \%$ CIs were then back-transformed to provide point estimates and $90 \%$ CIs for the test-to-reference ratio for the PK parameters $\mathrm{Cmax}, \mathrm{AUC}\left(0_{-} \tau\right), \mathrm{t}(1 / 2)$, and $\mathrm{CL} / \mathrm{F}$.

\section{In Vitro Metformin Transport Studies}

Effects of dolutegravir on metformin transports, including MATE1, MATE2-K, PMAT, and OCT3, were evaluated in vitro using recombinant expression systems with transport function confirmed by positive control inhibitors. Effects of dolutegravir on paracellular permeability were determined in confluent Madin-Darby canine kidney II and Caco-2 cell monolayers. Cellular uptake of metformin by the Caco-2 intestinal cell line was determined in the absence and presence of metformin (see Methods, Supplemental Digital Content, http://links.lww.com/QAI/A800 which describes the detailed methodologies used in the in vitro transport studies).

\section{RESULTS}

\section{Subject Demographics and Disposition}

Fifteen subjects were enrolled into cohort 1 and 14 subjects completed the study as planned; 1 subject was withdrawn from period 2 at the discretion of the investigator (difficulty obtaining blood samples). The majority of subjects in Cohort 1 were white (93\%), male $(73 \%)$, and had a mean age of 37.7 years. Fifteen subjects were enrolled into cohort 2 and 13 subjects completed as planned; 1 subject was withdrawn from period 2 at the discretion of the investigator (difficulty obtaining blood samples) and 1 subject was withdrawn from period 3 due to an $\mathrm{AE}$ (erythematous maculopapular rash). Subjects in cohort 2 were predominantly male (80\%), white (47\%) or African American (47\%), and had a mean age of 33.9 years.

\section{Pharmacokinetics}

The pharmacokinetics of metformin were altered in the presence of dolutegravir as shown by the increase in plasma exposure [Cmax and $\mathrm{AUC}(0-\tau)]$ and returned to baseline (period 1) 10 days after dolutegravir dosing was stopped (Fig. 1 and Table 1). The magnitude of the effect of dolutegravir on metformin PK was dependent on dolutegravir dose.

Metformin AUC $(0-\tau)$ and Cmax were increased by $79 \%$ and $66 \%$, respectively, when administered with dolutegravir 50 mg $\mathrm{q} 24 \mathrm{~h}$ and by $145 \%$ and $111 \%$, respectively, when administered with dolutegravir $50 \mathrm{mg}$ q12h (Table 1). This was accompanied by a decrease in CL/F of $44 \%$ and $59 \%$ when co-administered with dolutegravir $50 \mathrm{mg}$ q $24 \mathrm{~h}$ and $50 \mathrm{mg}$ q $12 \mathrm{~h}$, respectively (Table 1$)$. However, metformin $t(1 / 2)$ values were similar with and without co-administration with dolutegravir.

Dolutegravir PK parameters after co-administration of dolutegravir and metformin are presented in Table 2. The observed values of dolutegravir PK parameters after co-administration with metformin were comparable with dolutegravir administered alone based on historical data under fed 


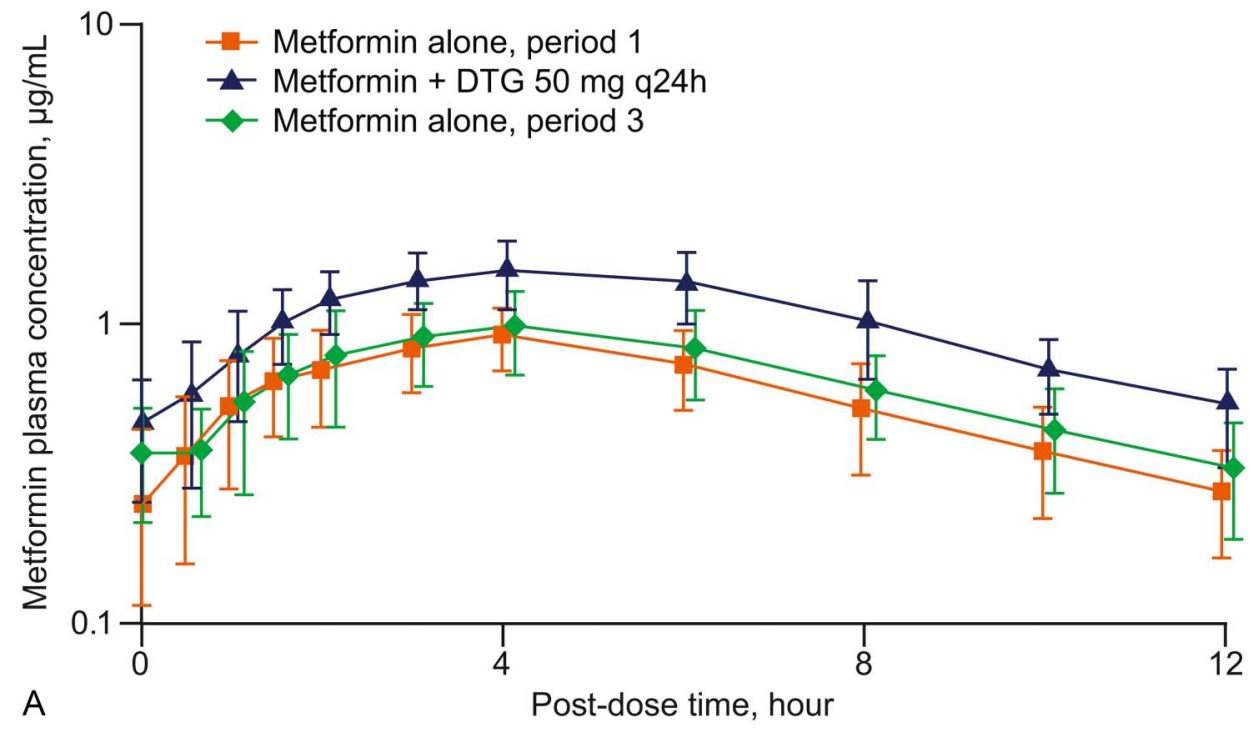

FIGURE 1. Mean plasma concentration-time profiles of metformin (500 mg q12h) administered with and without dolutegravir; (A) cohort 1 and (B) cohort 2. Period 1: metformin alone; period 2: metformin plus either dolutegravir $50 \mathrm{mg} \mathrm{q} 24 \mathrm{~h}$ (cohort 1) or dolutegravir $50 \mathrm{mg}$ q12h (cohort 2); period 3: metformin alone. DTG, dolutegravir; q12h, every 12 hours; q24h, every 24 hours.

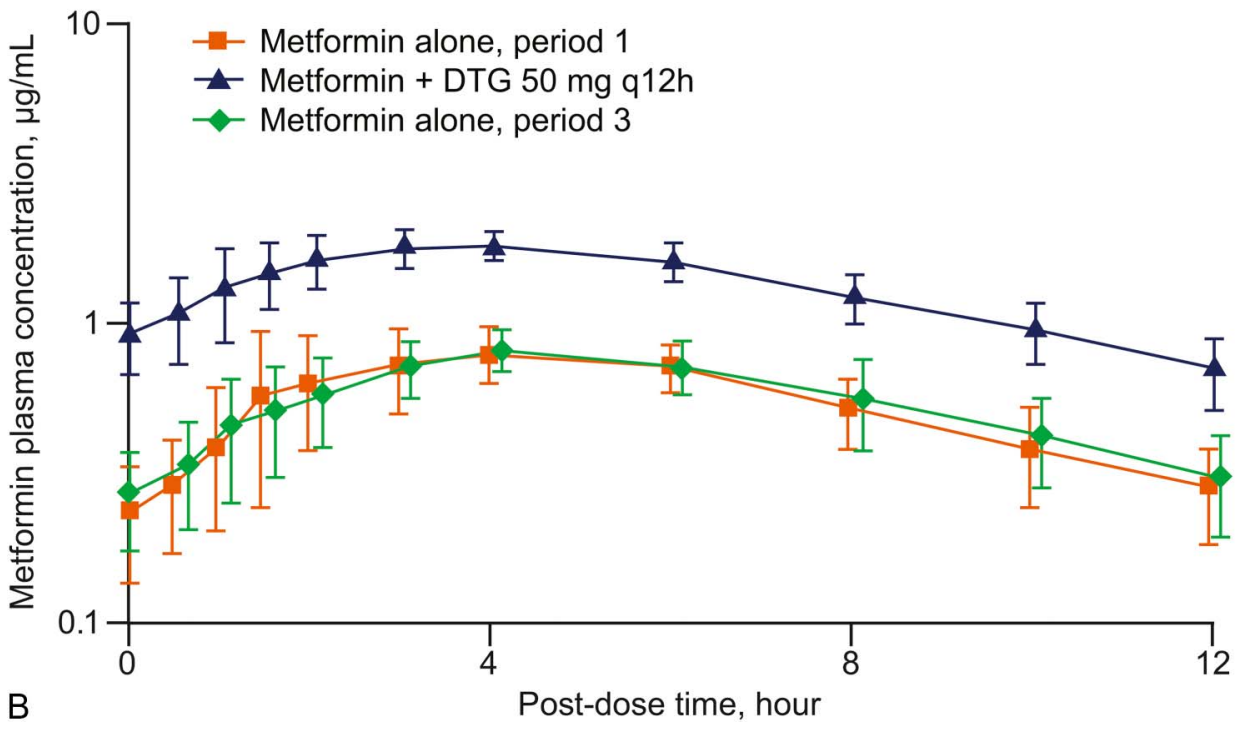

conditions. This is consistent with knowledge that metformin is not expected to affect the PK of dolutegravir.

\section{Safety}

All subjects received at least one dose of study medication and were included in the safety population for this study. Metformin was well tolerated when either administered alone (500 $\mathrm{mg} \mathrm{q} 12 \mathrm{~h}$ ) or when administered with dolutegravir (either $50 \mathrm{mg}$ q $24 \mathrm{~h}$ or $50 \mathrm{mg}$ q12h). There was no discernible pattern of AE frequency seen with respect to cohort or period. There were no AEs that were considered to be grade 3 or 4 (Division of AIDS), that resulted in death, or that were considered serious (nonfatal) reported during the study.

Overall, 26 AEs were reported by subjects in cohort 1, whereas 30 AEs were reported by subjects in cohort 2 (Table $3)$. Despite increased plasma concentrations of metformin when co-administered with dolutegravir (period 2), the frequency and severity of AEs in both cohorts were similar to those observed in period 1 (metformin alone). Additionally, there were more drug-related AEs reported during period 1 than during period 2 in both cohorts (Table 4). A single subject was withdrawn due to a drug-related $\mathrm{AE}$, erythematous maculopapular rash that occurred in period 3 (metformin alone), which began 2-3 days after dosing with dolutegravir was completed. One subject in cohort 2 had a grade $1 \mathrm{AE}$ of elevated ALT (133 U/L) in period 3, and this increase was considered potentially drug related, although the subject disclosed overeating fatty foods. The subject's ALT returned to normal limits, he completed the study, and he was discharged in good health. One subject in cohort 1 with a baseline glucose of $79 \mathrm{mg} / \mathrm{dL}$ experienced a grade $1 \mathrm{AE}$ of hypoglycemia in period 1 and period 2 that was considered drug related; however, the subject's symptoms (jitteriness and hand shaking) occurred twice in phase 1 with blood glucose values in the normal range ( 70 and $77 \mathrm{mg} / \mathrm{dL}$ ), and once on 
TABLE 1. Summary of Metformin PK Parameters

\begin{tabular}{|c|c|c|c|c|}
\hline \multirow[b]{3}{*}{ PK Parameter } & \multicolumn{4}{|c|}{ Cohort 1 (Dolutegravir 50 mg q24h) } \\
\hline & \multicolumn{3}{|c|}{ Geometric Mean (CV\%) } & \multirow{2}{*}{$\begin{array}{c}\text { GLS Mean Ratio }(90 \% \text { CI }) \\
\text { Period } 2 \text { vs Period } 1 \\
\end{array}$} \\
\hline & $\begin{array}{l}\text { Period } 1 \text { Metformin } \\
\text { Alone }(n=15)\end{array}$ & $\begin{array}{c}\text { Period 2 Metformin } \\
\text { Plus Dolutegravir }(n=14)\end{array}$ & $\begin{array}{l}\text { Period } 3 \text { Metformin } \\
\text { Alone }(\mathrm{n}=14)\end{array}$ & \\
\hline $\operatorname{AUC}(0-\tau), \mu \mathrm{g} \cdot \mathrm{h} / \mathrm{mL}$ & $6.82(26.5)$ & $12.2(23.1)$ & $7.55(28.1)$ & $1.79(1.65$ to 1.93$)$ \\
\hline $\mathrm{Cmax}, \mu \mathrm{g} / \mathrm{mL}$ & $0.932(26.2)$ & $1.55(22.6)$ & $0.979(31.1)$ & $1.66(1.53$ to 1.81$)$ \\
\hline $\operatorname{tmax}, \mathrm{h}^{*}$ & $4.00(1.00,6.00)$ & $4.00(1.00,6.03)$ & $4.00(1.00,6.00)$ & - \\
\hline \multirow{3}{*}{$\mathrm{t}(1 / 2), \mathrm{h}$} & \multicolumn{4}{|c|}{ Cohort 2 (Dolutegravir 50 mg q12h) } \\
\hline & \multicolumn{3}{|c|}{ Geometric Mean (CV\%) } & GLS Mean Ratio $(90 \%$ CI $)$ \\
\hline & $\begin{array}{l}\text { Period } 1 \text { Metformin } \\
\text { Alone }(n=15) \\
\end{array}$ & $\begin{array}{c}\text { Period } 2 \text { Metformin } \\
\text { Plus Dolutegravir }(n=14) \\
\end{array}$ & $\begin{array}{c}\text { Period } 3 \text { Metformin } \\
\text { Alone }(n=13) \\
\end{array}$ & Period 2 vs Period 1 \\
\hline $\mathrm{AUC}(0-\tau), \mu \mathrm{g} \cdot \mathrm{h} / \mathrm{mL}$ & $6.49(16.0)$ & $15.9(15.9)$ & $6.69(16.4)$ & $2.45(2.25$ to 2.66$)$ \\
\hline $\mathrm{t}(1 / 2), \mathrm{h}$ & $4.27(26.5) \dagger$ & 4.89 (19.6) & $4.48(24.3) \dagger$ & $1.114(1.00$ to 1.29$)$ \\
\hline
\end{tabular}

*tmax is reported as median (minimum, maximum).

$\uparrow$ PK parameter could not be calculated for one subject.

AUC $(0-\tau)$, area under the curve during a dosing interval; CI, confidence interval; CL/F, apparent clearance after oral dosing; Cmax, maximum concentration; GLS, geometric least squares; PK, pharmacokinetic; q12h, every 12 hours; q24h, every 24 hours; $t(1 / 2)$, half-life; tmax, time to maximum concentration.

day 1 of period 2 (first day of co-dosing), when his glucose was $55 \mathrm{mg} / \mathrm{dL}$ (normal range lower value $=70 \mathrm{mg} / \mathrm{dL}$ ), which responded quickly to oral glucose (juice), making attribution to glucose levels inconsistent. The subject was subsequently withdrawn from the study by the investigator due to repeated difficulty in obtaining blood samples for glucose monitoring.

No consistent, treatment related or clinically significant changes in mean or median hematology or clinical chemistry values were observed in the study. No clinically

TABLE 2. Summary of Dolutegravir PK Parameters

\begin{tabular}{|c|c|c|}
\hline & Cohort 1 & Cohort 2 \\
\hline $\begin{array}{l}\text { Dolutegravir PK } \\
\text { Parameter* }\end{array}$ & $\begin{array}{c}\text { Dolutegravir } 50 \mathrm{mg} \\
\text { q24h + Metformin } \\
500 \mathrm{mg} \text { q12h } \\
(\mathrm{N}=14)\end{array}$ & $\begin{array}{c}\text { Dolutegravir } 50 \mathrm{mg} \\
\text { q12h + Metformin } \\
500 \mathrm{mg} \mathrm{q12h} \\
(\mathrm{N}=14)\end{array}$ \\
\hline $\operatorname{AUC}(0-\tau), \mu g \cdot h / m L$ & $54.6(25.7)$ & $65.6(23.8)$ \\
\hline $\mathrm{Cmax}, \mu \mathrm{g} / \mathrm{mL}$ & $3.81(27.7)$ & $6.81(19.3)$ \\
\hline $\operatorname{tmax}, \mathrm{h}$ & $4.00(1.00,11.9)$ & $2.50(1.00,4.00)$ \\
\hline $\mathrm{CL} / \mathrm{F}, \mathrm{L} / \mathrm{h}$ & $0.914(26.7) \dagger$ & $0.762(23.8)$ \\
\hline $\mathrm{C} 0, \mu \mathrm{g} / \mathrm{mL}$ & $1.21(38.3)$ & $4.76(32.7)$ \\
\hline $\mathrm{C} \tau, \mu \mathrm{g} / \mathrm{mL}$ & $1.25(33.7)$ & $4.37(31.0)$ \\
\hline
\end{tabular}

*Data are presented as geometric mean $(\mathrm{CV} \%)$, except tmax, which is reported as median (minimum, maximum)

$\dagger$ PK parameter could not be calculated for one subject.

AUC $(0-\tau)$, area under the curve during a dosing interval; $\mathrm{C} \tau$, concentration at the end of the dosing interval; $\mathrm{C} 0$, predose concentration; $\mathrm{CL} / \mathrm{F}$, apparent clearance after oral dosing; Cmax, maximum concentration; $\mathrm{CV} \%$, coefficient of variation; $\mathrm{PK}$, pharmacokinetic; q12h, every 12 hours; q24h, every 24 hours; tmax, time to maximum concentration. significant changes in blood pressure or heart rate were observed during the study. Across both cohorts, mean serum creatinine levels were increased in period 2 at levels that are consistent with previous experience with dolutegravir (Table 4). ${ }^{13}$ In period 3 serum creatinine returned to levels similar to those observed in period 1 .

\section{In Vitro Metformin Transport Studies}

The in vitro transport studies showed that dolutegravir was not a clinically relevant inhibitor of PMAT (no inhibition $\leq 100 \mu \mathrm{M}$ ), OCT3 (no inhibition $\leq 10 \mu \mathrm{M} ; 10 \%-25 \%$ inhibition 30-100 $\mu \mathrm{M}$ ), MATE1 [half-maximal inhibitory concentration $\left.\left(\mathrm{IC}_{50}\right)=6.3 \pm 1.1 \mu \mathrm{M}\right]$, or MATE2-K $\left(\mathrm{IC}_{50}=\right.$ $24.8 \pm 4.7 \mu \mathrm{M})$. Paracellular permeability was not affected by dolutegravir in confluent cell monolayers. Likewise, metformin paracellular permeability and cellular uptake of metformin in the Caco-2 intestinal cell line were unaffected in the presence of dolutegravir (see Text 2 Results, Supplemental Digital Content, http://links.lww.com/QAI/A800 which describes findings in the in vitro transport studies; see Table 1, Supplemental Digital Content, http://links.lww.com/QAI/A800 which describes the findings on the effect of dolutegravir on [14C]metformin flux in Caco-2 cell monolayers; see Table 2, Supplemental Digital Content, http://links.lww.com/QAI/A800 which describes the findings on the effect of dolutegravir on [14C]metformin cellular uptake in Caco-2 cell monolayers; see Figure 1, Supplemental Digital Content, http://links.lww.com/QAI/A800 which describes the findings on the effect of dolutegravir on metformin transport by MATE1 and MATE2-K; see Figure 2, Supplemental Digital Content, http://links.lww.com/QAI/A800 which describes the 
TABLE 3. Summary of Drug-Related Adverse Events

\begin{tabular}{|c|c|c|c|c|c|c|}
\hline & \multicolumn{2}{|c|}{ Period 1 Metformin Alone } & \multicolumn{2}{|c|}{ Period 2 Metformin Plus Dolutegravir } & \multicolumn{2}{|c|}{ Period 3 Metformin Alone } \\
\hline & $\begin{array}{c}\text { Cohort 1* } \\
(N=15)\end{array}$ & $\begin{array}{c}\text { Cohort } 2 \dagger \\
(\mathbf{N}=15)\end{array}$ & $\begin{array}{l}\text { Cohort 1* } \\
(N=15)\end{array}$ & $\begin{array}{c}\text { Cohort 2† } \\
(\mathbf{N}=15)\end{array}$ & $\begin{array}{c}\text { Cohort 1* } \\
(N=14)\end{array}$ & $\begin{array}{c}\text { Cohort 2 } \dagger \\
(\mathrm{N}=14)\end{array}$ \\
\hline Any event $\$ \mathrm{n}(\%)$ & $7(47)$ & $2(13)$ & $2(13)$ & $5(33)$ & $1(7)$ & $2(14)$ \\
\hline Abdominal pain & $1(7)$ & & $1(7)$ & & 0 & \\
\hline Constipation & $2(13)$ & & 0 & & 0 & \\
\hline Abdominal discomfort & $1(7)$ & $1(7)$ & 0 & $1(7)$ & 0 & 0 \\
\hline Nausea & 0 & $1(7)$ & 0 & $3(20)$ & $1(7)$ & 0 \\
\hline Restless leg syndrome & $2(13)$ & & 0 & & 0 & \\
\hline Headache & 0 & $1(7)$ & $1(7)$ & 0 & 0 & 0 \\
\hline Hypoglycemia & $1(7)$ & & 0 & & 0 & \\
\hline Hyperhidrosis & 0 & 0 & 0 & $1(7)$ & 0 & 0 \\
\hline Erythematous maculopapular rash & 0 & 0 & 0 & 0 & 0 & $1(7)$ \\
\hline Dysuria & 0 & 0 & 0 & $1(7)$ & 0 & 0 \\
\hline
\end{tabular}

Adverse events are ordered by decreasing frequency in cohort 1 .

*Cohort 1 received dolutegravir $50 \mathrm{mg} \mathrm{q} 24 \mathrm{~h}$ in period 2.

$\dagger$ Cohort 2 received dolutegravir $50 \mathrm{mg} \mathrm{q} 12 \mathrm{~h}$ in period 2.

tSubjects with more than 1 drug-related adverse event are only counted once.

ALT, alanine aminotransferase; q12h, every 12 hours; q24h, every 24 hours.

findings on the effect of cimetidine on metformin transport by MATE1 and MATE2-K; see Figure 3, Supplemental Digital Content, http://links.lww.com/QAI/A800 which describes the findings on the effect of dolutegravir on MPP+ transport by PMAT and OCT3; and see Figure 4, Supplemental Digital Content, http://links.lww.com/QAI/A800 which describes the findings on the effect of dolutegravir on metformin transport by PMAT and OCT3).

\section{DISCUSSION}

Combination ART for the treatment of HIV infection is associated with a risk of developing T2DM, especially in subjects with signs of metabolic syndrome before initiating ART ${ }^{17}$ It is anticipated that antidiabetic medications, and in particular metformin, could be used concurrently with dolutegravir. Since dolutegravir is a clinically relevant inhibitor of OCT2 $\left(\mathrm{IC}_{50}=1.9 \mu \mathrm{M}\right),{ }^{14,18}$ a transporter involved in the renal elimination of metformin, it was important to evaluate the effect of dolutegravir on the PK, safety, and tolerability of metformin.

Since dolutegravir is a known inhibitor of OCT2, international labels contraindicate co-dosing of dolutegravir with OCT2 substrates that have a narrow therapeutic index, such as the antiarrhythmic agent dofetilide, due to the possible severe risk from increased drug levels when subjects are not monitored. Metformin is a drug that requires titration and has a very wide therapeutic window.

Consistent with inhibition of OCT2, co-administration of dolutegravir increased the exposure and decreased the

TABLE 4. Summary of Changes in Serum Creatinine

\begin{tabular}{|c|c|c|c|c|}
\hline \multirow[b]{2}{*}{ Period/Study Day } & \multicolumn{4}{|c|}{ Serum Creatinine, Mean (SD), $\mu \mathrm{mol} / \mathrm{L}$} \\
\hline & Cohort 1* & $\begin{array}{c}\text { Change From } \\
\text { Baseline (Predose) }\end{array}$ & Cohort 2* & $\begin{array}{c}\text { Change From } \\
\text { Baseline (Predose) }\end{array}$ \\
\hline Period $1, \uparrow$ day -1 (predose) & $68.36(12.71)$ & - & $78.97(16.53)$ & - \\
\hline Period $1, \dagger$ day 5 & 74.85 (11.99) & $5.89(4.31)$ & $80.74(16.32)$ & $1.77(9.57)$ \\
\hline Period $2, \div$ day 7 & $82.09(11.20)$ & $13.26(4.59)$ & $89.03(15.30)$ & $7.58(9.08)$ \\
\hline Period $3, \S$ day 10 & $72.61(12.59)$ & $3.79(6.68)$ & $78.20(13.43)$ & $-4.08(5.86)$ \\
\hline
\end{tabular}


clearance of metformin, an effect that was dose dependent. The magnitude to which dolutegravir increased the exposure to metformin was higher than anticipated $(5 \%)$ based on the $\mathrm{IC}_{50}$ values for OCT2 $(1.9 \mu \mathrm{M})$, dolutegravir unbound $\mathrm{Cmax}$ (twice daily $\mathrm{Cmax}=13 \mu \mathrm{M}$; free fraction, $\geq 1.1 \%$ ), and the fraction of metformin clearance mediated by active tubular secretion (0.67). ${ }^{19}$ Even taking dolutegravir's OCT2 inhibitory potency determined using tetraethylammonium as the substrate $(0.066 \mu \mathrm{M}),{ }^{18}$ the magnitude of the clinical metformin interaction is underpredicted by 2 -fold. Notably, the magnitude of the increase in metformin exposure in the presence of dolutegravir exceeded those observed for cimetidine and pyrimethamine, 2 prototypical inhibitors of renal OCT/MATE cationic transport. ${ }^{20-22}$ Given that $\mathrm{t}(1 / 2)$ values of metformin were not affected by co-administration of dolutegravir, and that the observed magnitude of the drugdrug interaction can be only partially explained by OCT2 inhibition, mechanisms other than inhibition of renal clearance of metformin are likely involved in the observed drug interaction. For this reason, the effect of dolutegravir on other transporters known to be involved in metformin absorption, distribution, and excretion (PMAT, OCT3, MATE1, and MATE2-K) was evaluated. ${ }^{8}$ Previous studies demonstrated that dolutegravir was not a clinically relevant inhibitor of OCT1 $(22 \%$ inhibition at $10 \mu \mathrm{M}),{ }^{14}$ and the present study showed that MATE1 inhibition $\left(\mathrm{IC}_{50}=6.3 \mu \mathrm{M}\right)$ also was not clinically relevant, ${ }^{23,24}$ whereas dolutegravir did not inhibit the other known metformin transporters. Consistent with the unchanged metformin tmax during period 2 of the present study, dolutegravir did not enhance metformin absorption through the paracellular pathway, and it did not affect metformin intestinal cell uptake, a process in which all the transport systems have not been reported in the literature. ${ }^{9}$ As such, the mechanisms of the dolutegravir-metformin drug-drug interaction include OCT2 and also other unidentified process(es).

This study demonstrated that, despite a significant increase in exposure, metformin was well tolerated when used in combination with dolutegravir. No pattern of $\mathrm{AE}$ frequency was seen with respect to cohort or period. The subject with the reported symptoms consistent with possible hypoglycemia had these episodes before the PK effect of codosing could be realized (day 1 of period 2, and earlier), and moreover, is inconsistent with the known $\mathrm{AE}$ profile and glucose-modulating effects of metformin. In the phase III clinical trials of dolutegravir, subjects entering the studies on metformin (or a metformin-containing product) were permitted to continue metformin therapy with appropriate monitoring. There were a total of 41 subjects who received dolutegravir and metformin (or a metformin-containing product) during phase III clinical trials of dolutegravir. Although these studies were not designed to evaluate the safety or PK impact of concomitant therapy with dolutegravir and metformin, no cases of drug-related hypoglycemia or lactic acidosis were reported in subjects receiving concomitant therapy.

Metformin has a wide therapeutic index and is not associated with a risk for hypoglycemia when administered at the recommended doses. Additionally, the primary safety concern with high metformin concentrations is lactic acidosis, which tends to occur in subjects with certain risk factors, most notably renal insufficiency (either intrinsic, including age-related decreases in renal function or from hypoperfusion secondary to sepsis, severe dehydration, etc.). There can also be an increased risk with either moderate liver disease or heavy ethanol use due to decreased metabolism of lactate. When metformin was considered the probable cause of lactic acidosis, the plasma concentrations were usually greater than $5 \mu \mathrm{g} / \mathrm{mL} .^{12}$ Of note, this plasma level is significantly higher than the Cmax achieved for subjects receiving twice-daily dolutegravir plus metformin in this study $(1.85 \mu \mathrm{g} / \mathrm{mL})$. Higher doses of metformin can also cause gastrointestinal intolerance, and dose titration is recommended to avoid these issues. Additive gastrointestinal intolerance would not be expected, because it was not observed in this study or the dolutegravir phase III program. The magnitude of the increase in metformin plasma PK by dolutegravir warrants monitoring of subjects' glucose levels when they are starting or stopping co-administration with metformin, and consideration of metformin dose adjustment based on glycemic control.

In conclusion, a dose adjustment in metformin should be considered when co-administered with dolutegravir, especially in subjects otherwise at risk for metformin toxicity. For subjects who start metformin therapy to treat T2DM while already on a dolutegravir-containing regimen for the treatment of HIV infection, treatment initiation and dose titration of metformin should be performed as suggested in the metformin product label. For subjects who start or stop a dolutegravir-containing regimen while on metformin therapy, the magnitude of the change in metformin plasma concentrations warrant reassessment of glycemic control and consideration of metformin dose adjustment.

\section{ACKNOWLEDGMENTS}

The authors wish to acknowledge the following individuals for editorial assistance during the development of this manuscript: John Busillo (GlaxoSmithKline) and Meredith MacPherson (MedThink SciCom).

\section{REFERENCES}

1. Song I, Chen S, Lou Y, et al. Pharmacokinetic (PK) and pharmacodynamic (PD) relationship of S/GSK1349572, a next generation integrase inhibitor (INI), in HIV-1 infected patients [WEPEB250]. Presented at: 5th IAS Conference on HIV Pathogenesis and Treatment and Prevention; 2009; Cape Town, South Africa.

2. Min S, Song I, Borland J, et al. Pharmacokinetics and safety of S/GSK1349572, a next-generation HIV integrase inhibitor, in healthy volunteers. Antimicrob Agents Chemother. 2010;54:254-258.

3. Raffi F, Jaeger H, Quiros-Roldan E, et al. Once-daily dolutegravir versus twice-daily raltegravir in antiretroviral-naive adults with HIV-1 infection (SPRING-2 study): 96 week results from a randomised, double-blind, non-inferiority trial. Lancet Infect Dis. 2013;13:927-935.

4. Walmsley SL, Antela A, Clumeck N, et al. Dolutegravir plus abacavirlamivudine for the treatment of HIV-1 infection. N Engl J Med. 2013; 369:1807-1818.

5. Cahn P, Pozniak AL, Mingrone $\mathrm{H}$, et al. Dolutegravir versus raltegravir in antiretroviral-experienced, integrase-inhibitor-naive adults with HIV: 
week 48 results from the randomised, double-blind, non-inferiority SAILING study. Lancet. 2013;382:700-708.

6. Castagna A, Maggiolo F, Penco G, et al; for the VIKING-3 Study Group. Dolutegravir in antiretroviral-experienced patients with raltegravirand/or elvitegravir-resistant HIV-1: 24-week results of the phase III VIKING-3 study. J Infect Dis. 2014;210:354-362.

7. Rojas LB, Gomes MB. Metformin: an old but still the best treatment option for type 2 diabetes. Diabetol Metab Syndr. 2013;5:6.

8. Graham GG, Punt J, Aroroa M, et al. Clinical pharmacokinetics of metformin. Clin Pharmacokinet. 2011;50:81-98.

9. Proctor WR, Bourdet DL, Thakker DR. Mechanisms underlying saturable intestinal absorption of metformin. Drug Metab Dispos. 2008;36:1650-1658.

10. Gong L, Goswami S, Giacomini KM, et al. Metformin pathways: pharmacokinetics and pharmacodynamics. Pharmacogenet Genomics. 2012;22:820-827.

11. Christiansen M, Pedersen R, Stage T, et al. A gene-gene interaction between polymorphisms in the OCT2 and MATE1 genes influences the renal clearance of metformin. Pharmacogenet Genomics. 2013;23:526-534.

12. Glucophage [Package Insert]. 2009. Available at: http://packageinserts. bms.com/pi/pi_glucophage_xr.pdf. Accessed December 2014.

13. Tivicay [Package Insert]. 2014. Available at: http://www.viivhealthcare. com/media/58599/us_tivicay.pdf. Accessed December 2014.

14. Reese MJ, Savina PM, Generaux GT, et al. In vitro investigations into the roles of drug transporters and metabolizing enzymes in the disposition and drug interactions of dolutegravir, a HIV integrase inhibitor. Drug Metab Dispos. 2013;41:353-361.

15. Koteff J, Borland J, Chen S, et al. A phase 1 study to evaluate the effect of dolutegravir on renal function via measurement of iohexol and paraaminohippurate clearance in healthy subjects. $\mathrm{Br} J$ Clin Pharmacol. 2013;75:990-996.

16. Division of AIDS Table for Grading the Severity of Adult and Pediatric Adverse Events. Version 1.0. Available at: http://rsc.tech-res.com/
Document/safetyandpharmacovigilance/Table_for_Grading_Severity_ of_Adult_Pediatric_Adverse_Events.pdf. Accessed January 2014 .

17. Wand H, Calmy A, Carey DL, et al. Metabolic syndrome, cardiovascular disease and type 2 diabetes mellitus after initiation of antiretroviral therapy in HIV infection. AIDS. 2007;21:2445-2453.

18. Lepist EI, Zhang X, Hao J, et al. Contribution of the organic anion transporter OAT2 to the renal active tubular secretion of creatinine and mechanism for serum creatinine elevations caused by cobicistat. Kidney Int. 2014;86:350-357.

19. Zamek-Gliszczynski MJ, Lee CA, Poirier A, et al. ITC recommendations for transporter kinetic parameter estimation and translational modeling of transport-mediated PK and DDIs in humans. Clin Pharmacol Ther. 2013; 94:64-79.

20. Tsuda M, Terada T, Ueba M, et al. Involvement of human multidrug and toxin extrusion 1 in the drug interaction between cimetidine and metformin in renal epithelial cells. $J$ Pharmacol Exp Ther. 2009;329:185-191.

21. Somogyi A, Stockley C, Keal J, et al. Reduction of metformin renal tubular secretion by cimetidine in man. Br J Clin Pharmacol. 1987;23: 545-551.

22. Kusuhara $\mathrm{H}$, Ito $\mathrm{S}$, Kumagai $\mathrm{Y}$, et al. Effects of a MATE protein inhibitor, pyrimethamine, on the renal elimination of metformin at oral microdose and at therapeutic dose in healthy subjects. Clin Pharmacol Ther. 2011;89:837-844.

23. US Department of Health and Human Services, Food and Drug Administration. Guidance for Industry: Drug Interaction Studies-Study Design, Data Analysis, Implications for Dosing, and Labeling Recommendations. Draft Guidance. Available at: http://www.fda.gov/downloads/Drugs/ GuidanceComplianceRegulatoryInformation/Guidances/UCM292362.pdf. Accessed April 21, 2015.

24. European Medicines Agency, Committee for Human Medicinal Products. Guideline on the Investigation of Drug Interactions (Final). Available at: http://www.ema.europa.eu/docs/en_GB/document_library/Scientific_ guideline/2012/07/WC500129606.pdf. Accessed April 21, 2015. 